\title{
Comportamiento Térmico de fachada ventilada opaca en clima cálido seco extremo
}

\section{Thermal Behavior of opaque ventilated facade in hot dry climate}

\author{
SOTELO-SALAS, Cristina†*, ESPARZA-LÓPEZ, Carlos Javier y ESCOBAR-DEL POZO, Carlos \\ Universidad de Colima, Facultad de Arquitectura y Diseño
}

ID $1^{\text {er }}$ Autor: Cristina, Sotelo-Salas / ORC ID: 0000-0001-8685-4290, CVU CONACYT ID: 358418

ID $1^{\mathrm{er}}$ Coautor: Carlos, Esparza-López / ORC ID: 0000-0003-0058-5072, CVU CONACYT ID: 335287

ID $2^{\text {do }}$ Coautor: Carlos, Escobar-Del Pozo / ORC ID: 0000-0002-8732-8791, CVU CONACYT ID: 43347

DOI: $10.35429 / J A D .2019 .10 .3 .20 .28$

Recibido 22 de Octubre, 2018; Aceptado 16 de Diciembre, 2018

\begin{abstract}
Resumen
El consumo energético atribuido a las edificaciones asciende a un $30 \%$ a nivel global. Dicho consumo es aún mayor en edificaciones que carecen de adecuación climática. En climas cálidos, los dispositivos de control solar protegen de las ganancias térmicas por radiación solar directa como estrategia para evitar el sobrecalentamiento en edificaciones. Las fachadas ventiladas permiten incrementar la eficiencia energética de dichos dispositivos al hacer uso del enfriamiento convectivo producto del diferencial de temperatura y presión al interior de la fachada ventilada. La presente investigación muestra el comportamiento térmico de una fachada ventilada opaca a través de modelación numérica en el programa de dinámica de fluidos computacional ANSYS Fluent ${ }^{\circledR}$ en un clima cálido seco extremo. El objetivo del estudio fue analizar el efecto de una fachada ventilada sobre el desempeño térmico del muro inmediato al espacio habitable como sistema pasivo de enfriamiento convectivo. Las variables analizadas fueron el espesor de la cavidad de aire, la temperatura y la velocidad de viento del ambiente exterior, así como su afectación en la temperatura superficial del muro adyacente al espacio habitable. Los resultados muestran una disminución en la temperatura superficial del muro, lo cual reduce la carga de enfriamiento total de la edificación.
\end{abstract}

Fachada ventilada opaca, Doble fachada, Dinámica de fluidos computacional, Ventilación natural

\begin{abstract}
Energy consumption attributed to buildings amounts to approximately $30 \%$ globally. This consumption is even greater in buildings that lack climate adaptation strategies. In warm climates, solar control devices protect from thermal gains by direct solar radiation as a strategy to avoid overheating in buildings. Ventilated facades increase the energy efficiency of said devices when harnessing the convective cooling produced by the temperature and pressure differential inside the ventilated facade. The present research shows the thermal behaviour of an opaque ventilated facade through numerical modelling in ANSYS Fluent ${ }^{\circledR}$ computational fluid dynamics software in an extreme hot dry climate. The objective of the study was to analyze the effect of a ventilated facade on the thermal performance of the wall immediately adjacent to the living space as a passive convective cooling system. The variables analyzed were thickness of the air cavity, ambient temperature and wind speed of the external environment, as well as its effect on the surface temperature of the wall adjacent to the living space. The results show a decrease in the surface temperature of the wall, which reduces the total cooling load of the building.
\end{abstract}

Opaque ventilated facade, Opaque double skin facade, Computational fluid dynamics, Natural ventilation

Citación: SOTELO-SALAS, Cristina, ESPARZA-LÓPEZ, Carlos Javier y ESCOBAR-DEL POZO, Carlos. Comportamiento Térmico de fachada ventilada opaca en clima cálido seco extremo. Revista de Arquitectura y Diseño. 2019, 3-10: 20-28

*Correspondencia al Autor (Correo Electrónico: csotelo1@ucol.mx)

$\dagger$ Investigador contribuyendo como primer autor. 


\section{Introducción}

En la actualidad, la tendencia de las edificaciones en las ciudades es dar mayor peso a lo vertical sobre lo horizontal con la finalidad de aumentar la densidad poblacional en las mismas y solventar los problemas de la urbanización expansionista pasada. Lo anterior conlleva a modificar los porcentajes previstos de injerencia de los componentes de la envolvente arquitectónica sobre el espacio interior. Antes, con edificaciones horizontales, la azotea tenía un papel predominante sobre las condiciones interiores en lugares intertropicales, incluso alcanzado hasta el $65 \%$ de la transferencia del calor hacia el espacio (Esparza-López, Gómez-Amador, GómezAzpeitia, González-Cruz y Escobar, 2015). Al modificar las proporciones de las edificaciones e incrementar el área de fachada al verticalizar la edificación, la tasa de intercambio térmico se modifica incrementando sobre las fachadas y disminuyendo en las azoteas.

Existen diversos dispositivos y sistemas de climatización que se utilizan sobre las fachadas para reducir y amortiguar el intercambio a través de estas (Halawa et al., 2018; Shameri, Alghoul, Sopian, Zain y Elayeb, 2011). Las fachadas dobles son dispositivos sobrepuestos a las fachadas que permiten mitigar o amortiguar el intercambio de energía entre el interior y el exterior (Barbosa e Ip, 2014). Dependiendo su objetivo, diseño y construcción, pueden permitir el paso de luz y ventilación, bloquear por completo la radiación solar y el aire, buscar la interconexión entre niveles, incrementar la privacidad o permitir la visibilidad del espacio (Halawa et al., 2018).

La selección de las características idóneas para el diseño y construcción de una doble fachada está en función de las estrategias de climatización que se pretenden utilizar en un lugar y clima determinado. Al ser elementos del diseño que se encuentran expuestos al exterior, las variables que se modifican con su incorporación son principalmente la radiación solar y la ventilación (Shameri et al., 2011).

Según Barbosa e Ip (2014) los parámetros de selección de las características se pueden dividir principalmente en factores de la propia fachada, del edificio y del sitio.
Dentro de los factores de la propia fachada encontramos la profundidad de la cavidad de aire, sombreado, superficie de vidrio, estructura y aberturas. De los factores del edificio se encuentran los materiales de la envolvente, la tasa de aberturas en vanos y transparencias; la altura de la cavidad y el número de niveles del edificio. Finalmente, para los parámetros del sitio, coincide con lo mencionado en Shameri et al., (2011) respecto a las principales estrategias a utilizar ya que alude a la radiación solar y el viento como actores importantes en su determinación.

Ghaffarianhoseini, Ghaffarianhoseini, Berardi, Tookey, Li y Kariminia (2016) inicialmente clasifican a las fachadas dobles como ventiladas o no ventiladas. Las fachadas no ventiladas se encuentran relativamente herméticas reduciendo aún más la transferencia de calor entre el interior y el exterior. Este tipo de fachadas son ideales para amortiguar las diferencias entre las temperaturas. Las fachadas dobles ventiladas permiten la circulación de aire a través de ellas, promoviendo la renovación de aire y, por ende, el intercambio de calor entre los espacios.

Las fachadas dobles, ya sean ventiladas o no, se pueden dividir en opacas, translúcidas y transparentes en función del material con el que están construidas que permite el paso de luz y visibilidad hacia el interior del espacio. Empero pueden cumplir otros objetivos más allá del sistema constructivo, e.g., la transferencia de calor, ya que, en función de la transparencia, es la cantidad de radiación que penetra al interior del espacio.

En función del efecto de flotabilidad del aire dentro de la cavidad (Sánchez, Rolando, Sant y Ayuso, 2016) o de la disipación de calor por convección en la totalidad de la fachada se puede definir si la fachada es de juntas abiertas o cerradas (Ibáñez-Puy, Vidaurre-Arbizu, Sacristán-Fernández y Martín-Gómez, 2017). Si la temperatura del aire exterior es inferior a la temperatura de la cavidad y lo que se busca es disipar el calor acumulado en la cavidad de aire, se puede optar por una fachada de juntas abiertas que permite el paso del aire a lo largo de la altura de la fachada. Un ejemplo de este tipo de formas son las celosías. 
Por otro lado, si se busca de bloqueo de la temperatura radiante de la doble fachada o complementar el dispositivo con otro tipo de estrategia, se puede elegir por una doble fachada de juntas cerradas.

La doble fachada de juntas cerradas no permite el paso del aire en el cuerpo de la estructura, dejando aberturas solo en la parte inferior y superior de la misma (Figura 1). Esto fomenta el fenómeno de flotabilidad y de convección natural del aire dentro de la cavidad.

Souza, Souza y Rodrigues (2018) demuestran la efectividad en la reducción de la temperatura tanto en la cavidad como en el espacio interior al utilizar fachadas dobles opacas ventiladas de juntas cerradas. Sin embargo, es importante señalar que la utilización de esta no implica un efecto de enfriamiento dentro del espacio, sino de reducción de las ganancias provenientes del exterior.

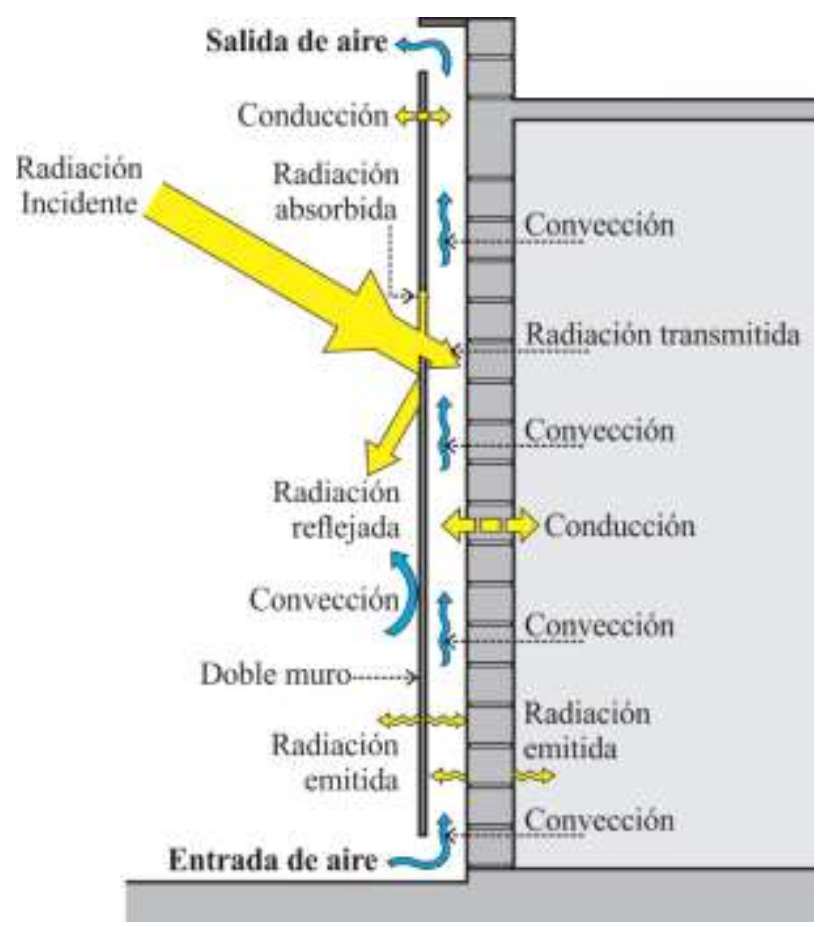

Figura 1 Componentes de una fachada ventilada y mecanismos de transferencia de calor que intervienen en su comportamiento térmico

Fuente: Elaboración propia con base en Barbosa, Ip y Southall (2015)
El objetivo de este estudio es analizar el comportamiento térmico de una fachada ventilada opaca en una edificación de un nivel en clima cálido seco extremo para determinar su efecto sobre la temperatura superficial del muro interior en contacto con el espacio habitable, de tal manera que permita definir lineamientos de diseño para la aplicación de este dispositivo de protección solar en edificaciones nuevas y existentes con la finalidad de disminuir las ganancias por radiación solar directa y potenciar el enfriamiento por convección en la cavidad de aire.

Se describe la metodología a partir de simulación en dinámica de fluidos computacional (CFD por sus siglas en inglés), así como el proceso de validación de dichos modelos. Se muestra el efecto de variables ambientales que impactan en el comportamiento térmico de la fachada ventilada opaca, así como la influencia de la distancia entre la doble fachada y el muro en contacto con el espacio interior.

Finalmente se discuten los resultados para determinar el caso con mejor desempeño, es decir, aquel que muestre una diferencia de temperatura mayor con respecto al caso base y determinar la configuración geométrica más apropiada según su interacción con las variables de temperatura de bulbo seco exterior (TBS) y la velocidad de viento del ambiente exterior.

\section{Metodología}

\section{Simulación CFD: caso de referencia}

El estudio se realiza en el programa de dinámica fluidos computacional ANSYS Fluent ${ }^{\circledR} 15$, el caso de referencia para la realización del análisis es una edificación de un solo nivel, cuyas dimensiones en planta son de $3 \mathrm{~m} \times 3 \mathrm{~m}$, con $3 \mathrm{~m}$ de altura. Las variables independientes son la temperatura de bulbo seco exterior, velocidad de viento, y el ancho de la cavidad de aire; las variables dependientes son la temperatura al centro de la cavidad de aire, la temperatura superficial del muro sombreado por la doble fachada, y la velocidad de viento al centro de la cavidad de aire. 
En la ciudad de Mexicali se tienen registros de temperatura máxima extrema de 45 ${ }^{\circ} \mathrm{C}$ y un récord histórico de $54{ }^{\circ} \mathrm{C}$ (Bojórquez, Gómez-Azpeitia, García-Cueto, Ruiz-Torres, y Luna, 2010), por lo cual se considera que es un sitio representativo del clima cálido seco extremo donde propone el estudio.

Mexicali se ubica al noreste del estado de Baja California, en la frontera norte de México, en latitud de $32^{\circ} 39^{\prime} 48^{\prime \prime} \mathrm{N}$ y longitud de $115^{\circ} 28^{\prime} 04^{\prime \prime} \mathrm{O}$. La edificación utilizada como referencia se caracteriza con la tipología de construcción utilizada en Mexicali, block común de concreto de $0.12 \mathrm{~m}$ x $0.2 \mathrm{~m}$ x $0.4 \mathrm{~m}$, para vivienda de interés social (Gutiérrez, Romero y Sotelo, 2014).

Se consideran dos valores de temperatura de bulbo seco, la máxima media del mes de julio, considerado el mes crítico del período cálido que abarca los meses de mayo a octubre, obtenidos del archivo climático de la estación meteorológica del aeropuerto de Mexicali, que analiza valores de 2000-2009, con $47.1{ }^{\circ} \mathrm{C}$ como temperatura máxima mensual, y $35{ }^{\circ} \mathrm{C}$ como temperatura media mensual.

Adicionalmente, se utilizaron valores de velocidad de viento de $1.5,4$ y $6.5 \mathrm{~m} / \mathrm{s}$, la mínima, media y máxima respectivamente.

Respecto a la configuración geométrica de la fachada ventilada, estudios previos reportan que los efectos de enfriamiento se manifiestan a partir de $0.07 \mathrm{~m}$, y que su comportamiento térmico se estabiliza desde los 0.35 m (Ibáñez-Puy et al., 2017), por tanto, en este estudio se manejan intervalos regulares de $0.1 \mathrm{~m}$ en un rango de $0.1 \mathrm{~m}$ a $0.5 \mathrm{~m}$. Los escenarios de simulación y su nomenclatura se describen en la tabla 1.

\begin{tabular}{|c|c|c|c|}
\hline Caso & $\begin{array}{l}\text { Ancho } \\
\text { cavidad de } \\
\text { aire }[\mathrm{m}]\end{array}$ & $\begin{array}{l}\text { Temperatura de } \\
\text { Bulbo Seco } \\
\text { exterior }\left[{ }^{\circ} \mathrm{C}\right]\end{array}$ & $\begin{array}{l}\text { Velocidad de } \\
\text { viento }[\mathrm{m} / \mathrm{s}]\end{array}$ \\
\hline C1 & 0.1 & 47.1 & 6.5 \\
\hline $\mathrm{C} 2$ & 0.1 & 47.1 & 4 \\
\hline C3 & 0.1 & 47.1 & 1.5 \\
\hline $\mathrm{C} 4$ & 0.1 & 35 & 6.5 \\
\hline $\mathrm{C} 5$ & 0.1 & 35 & 4 \\
\hline C6 & 0.1 & 35 & 1.5 \\
\hline $\mathrm{C7}$ & 0.2 & 47.1 & 6.5 \\
\hline $\mathrm{C} 8$ & 0.2 & 47.1 & 4 \\
\hline C9 & 0.2 & 47.1 & 1.5 \\
\hline $\mathrm{C} 10$ & 0.2 & 35 & 6.5 \\
\hline C11 & 0.2 & 35 & 4 \\
\hline $\mathrm{C} 12$ & 0.2 & 35 & 1.5 \\
\hline $\mathrm{C} 13$ & 0.3 & 47.1 & 6.5 \\
\hline C14 & 0.3 & 47.1 & 4 \\
\hline $\mathrm{C} 15$ & 0.3 & 47.1 & 1.5 \\
\hline C16 & 0.3 & 35 & 6.5 \\
\hline $\mathrm{C} 17$ & 0.3 & 35 & 4 \\
\hline C18 & 0.3 & 35 & 1.5 \\
\hline C19 & 0.4 & 47.1 & 6.5 \\
\hline $\mathrm{C} 20$ & 0.4 & 47.1 & 4 \\
\hline $\mathrm{C} 21$ & 0.4 & 47.1 & 1.5 \\
\hline $\mathrm{C} 22$ & 0.4 & 35 & 6.5 \\
\hline $\mathrm{C} 23$ & 0.4 & 35 & 4 \\
\hline $\mathrm{C} 24$ & 0.4 & 35 & 1.5 \\
\hline C25 & 0.5 & 47.1 & 6.5 \\
\hline C26 & 0.5 & 47.1 & 4 \\
\hline C27 & 0.5 & 47.1 & 1.5 \\
\hline C28 & 0.5 & 35 & 6.5 \\
\hline C29 & 0.5 & 35 & 4 \\
\hline C30 & 0.5 & 35 & 1.5 \\
\hline
\end{tabular}

Tabla 1 Escenarios de simulación

Se contrastan los resultados de los escenarios de simulación contra un caso base (CB), es decir, un muro orientado al sur completamente expuesto a la radiación solar directa.

El doble muro que conforma a la fachada ventilada se ubica al sur y es el único muro en el estudio donde se implementa el dispositivo en el modelo virtual.

2. Geometría computacional y análisis de malla

Se realizó un modelo computacional con dimensiones de $15 \mathrm{~m}$ x $20 \mathrm{~m}$ x $10 \mathrm{~m}$ de altura (Figura 2). La geometría se realizó con el programa ANSYS DesignModeler®, y la malla se generó con ANSYS Meshing®, donde el tamaño de la malla se determinó a partir de un análisis de sensibilidad, de tal manera que el tamaño de la malla no afectara la simulación del fenómeno estudiado. 


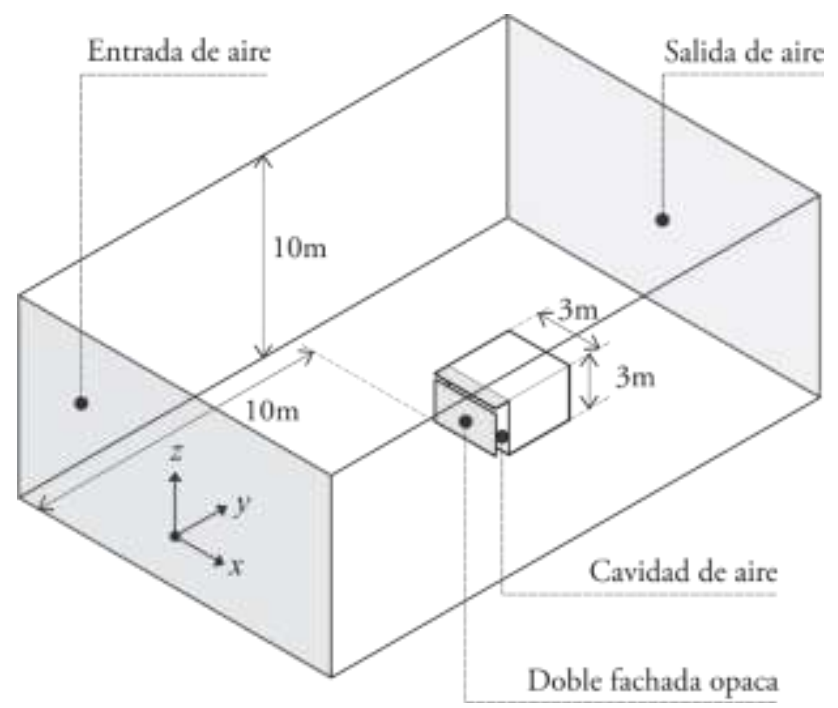

Figura 2 Geometría del modelo analizado

Se consideraron tres tamaños de malla denominados malla burda con 1,159,948 elementos, malla media con $1,870,759$ elementos y malla fina con 2,611,906 elementos y se tomaron 27 puntos a lo largo del eje z para la comparación (Gráfica 1). Entre la malla burda y la malla media se presentó un error $0.24 \%$ y entre la malla media y la malla fina se encontró un error de $0.12 \%$. Debido a la concordancia mostrada entre las mallas analizadas, se determina que la malla media provee suficiente precisión a menor costo computacional, y se selecciona para realizar el estudio propuesto.

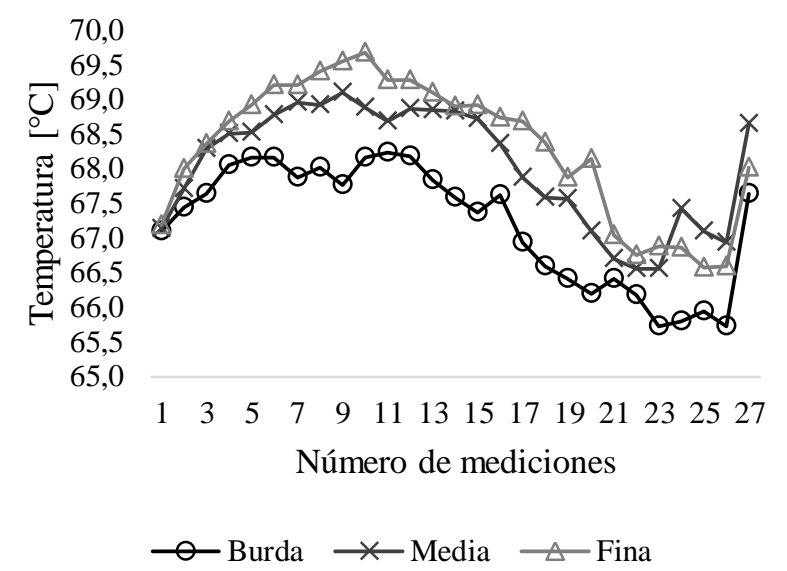

Gráfica 1 Análisis de sensibilidad de malla

\section{Condiciones de frontera}

La entrada de aire se configura con los parámetros físicos del ambiente exterior según cada escenario de simulación (Tabla 1), con un perfil de velocidad uniforme $\left(=U_{\infty}\right)$ de acuerdo con el rango de valores seleccionado.
Se utilizó la metodología señalada por Montazeri, Blocken y Hensen (2015) para calcular la energía cinética turbulenta $k$ a partir de $U_{\infty}$ y de la intensidad de la turbulencia, $I$, con valor de $10 \%$ para aplicaciones prácticas de flujo de viento en la capa límite atmosférica, con la ecuación (1); la escala de longitud de turbulencia, $l$, se calculó a partir del diámetro hidráulico, $D_{H}$, del dominio computacional, con la ecuación (2); la tasa de disipación de turbulencia, $\varepsilon$, se calculó a partir de la ecuación (3), donde $C_{\mu}$ es una constante con valor aproximado de 0.09 .

$k=\left(U_{\infty} \cdot I\right)^{2}$

$l=0.07 D_{H}$

$\varepsilon=C_{\mu}^{3 / 4}\left(\frac{k^{3 / 2}}{l}\right)$

Las superficies que representan a la edificación de un nivel se caracterizaron como muros de block común de concreto de $0.12 \mathrm{~m}$ con una conductividad térmica de $1.013 \mathrm{~W} / \mathrm{m}$ $\mathrm{K}$. El doble muro que forma la cavidad de aire de la fachada ventilada se caracterizó con tablero de cemento USG Durock ${ }^{\circledR}$ de $0.0127 \mathrm{~m}$ de espesor con una conductividad térmica de $0.099 \mathrm{~W} / \mathrm{m} \mathrm{K}$.

Se empleó el modelo de radiación Surface-to-Surface, con los valores para radiación solar directa de $879.2 \mathrm{~W} / \mathrm{m}^{2}$, radiación solar difusa en superficie vertical de $77.5 \mathrm{~W} / \mathrm{m}^{2}$ y $119.22 \mathrm{~W} / \mathrm{m}^{2}$ en superficie horizontal.

\section{Validación}

Para validar las simulaciones realizadas se realizó el análisis de concordancia con el índice propuesto por Willmott (1981), donde se comparan las mediciones obtenidas por experimentación con las mediciones simuladas.

Se utilizaron las mediciones de campo en un módulo experimental ubicado en la Facultad de Arquitectura y Diseño de la Universidad Autónoma de Baja California, campus Mexicali, a una latitud de $32^{\circ} 37^{\prime} 57^{\prime \prime} \mathrm{N}$ y una longitud de $115^{\circ} 26^{\prime} 36^{\prime \prime} \mathrm{O}$, y a una altura sobre el nivel del mar de $5 \mathrm{~m}$ a nivel de piso. 
Sin embargo, debido a que los módulos se ubican en la cubierta del edificio, se adicionan $9.92 \mathrm{~m}$ para un total de $14.92 \mathrm{~m}$ de altura sobre el nivel del mar en la ubicación de los módulos.

El análisis muestra un error de $1.9 \%$ para las mediciones al centro de la cavidad de aire y de $1.52 \%$ para las mediciones de temperatura superficial.

\section{Resultados}

\section{Simulaciones CFD: análisis de parámetros físicos}

Los parámetros físicos que se utilizan como variables independientes para este estudio son la temperatura de bulbo seco exterior y la velocidad de viento, en esta sección se describen los efectos de ambas, así como la relación de estas con la configuración geométrica de la fachada ventilada, determinada por el ancho de la cavidad de aire.

\section{Efecto de la temperatura de bulbo seco de entrada}

Se indica en la tabla 2 la temperatura media para cada caso con temperatura de bulbo seco exterior de $47.1{ }^{\circ} \mathrm{C}$, la comparación con la temperatura media del caso base bajo las mismas condiciones (CB47), y la diferencia de cada escenario con respecto al CB47.

En la tabla 3 se muestra la temperatura media para cada caso con TBS exterior de 35 ${ }^{\circ} \mathrm{C}$, la comparación con la temperatura media del caso base bajo las mismas condiciones y la diferencia de estas con el caso base respectivo (CB35).

Se observa que a pesar de la diferencia de las condiciones de TBS, el efecto de sombreado y convección natural sobre el muro sur es similar, con variaciones de 0.2 a $1.5{ }^{\circ} \mathrm{C}$ en la reducción en la temperatura superficial del muro en contacto con el espacio habitable.

\begin{tabular}{|l|r|r|}
\hline \multicolumn{1}{|c}{ Caso } & $\begin{array}{c}\text { Temperatura } \\
\text { superficial media } \\
{\left[{ }^{\circ} \mathbf{C}\right]}\end{array}$ & $\begin{array}{c}\text { Reducción de } \\
\text { temperatura }\left[{ }^{\circ} \mathbf{C}\right]\end{array}$ \\
\hline CB47 & 67.81 & N/A \\
\hline C1 & 53.9 & 13.9 \\
\hline C2 & 55.6 & 12.2 \\
\hline C3 & 60.4 & 7.41 \\
\hline C7 & 53.6 & 14.2 \\
\hline C8 & 56 & 11.8 \\
\hline C9 & 60.6 & 7.23 \\
\hline C13 & $\mathbf{5 3 . 1}$ & $\mathbf{1 4 . 8}$ \\
\hline C14 & 55.6 & 12.2 \\
\hline C15 & 60.9 & 6.94 \\
\hline C19 & 53.5 & 14.3 \\
\hline C20 & 55.9 & 11.9 \\
\hline C21 & 60.8 & 7 \\
\hline C25 & 53.3 & 14.5 \\
\hline C26 & 55.8 & 12 \\
\hline C27 & 59.8 & 7.97 \\
\hline
\end{tabular}

Tabla 2 Temperatura superficial media en muro de block común de concreto y la reducción respecto al caso base

\begin{tabular}{|l|r|r|}
\hline Caso & $\begin{array}{c}\text { Temperatura } \\
\text { superficial media } \\
{\left[{ }^{\circ} \mathbf{C}\right]}\end{array}$ & $\begin{array}{c}\text { Reducción de } \\
\text { temperatura }\left[{ }^{\circ} \mathbf{C}\right]\end{array}$ \\
\hline CB35 & 56.18 & N/A \\
\hline C4 & 43.4 & 12.8 \\
\hline C5 & 45.5 & 10.7 \\
\hline C6 & 49.4 & 6.73 \\
\hline C10 & 42.3 & 13.9 \\
\hline C11 & 44.6 & 11.6 \\
\hline C12 & 49.5 & 6.71 \\
\hline C16 & $\mathbf{4 1 . 8}$ & $\mathbf{1 4 . 3}$ \\
\hline C17 & 44.4 & 11.8 \\
\hline C18 & 49.8 & 6.42 \\
\hline C22 & 42 & 14.1 \\
\hline C23 & 44.6 & 11.6 \\
\hline C24 & 49.7 & 6.53 \\
\hline C28 & 42.1 & 14.1 \\
\hline C29 & 44.5 & 11.6 \\
\hline C30 & 48.8 & 7.42 \\
\hline
\end{tabular}

Tabla 3 Temperatura superficial media en muro de block común de concreto y la reducción respecto al caso base

\section{Efecto de la velocidad de aire de entrada}

Los casos C1, C7, C13, C19, C25, con TBS exterior de $47.1{ }^{\circ} \mathrm{C}$ y velocidad de viento de 6.5 $\mathrm{m} / \mathrm{s}$, muestran mayor reducción de temperatura media al interior de la cavidad de aire y en la superficie del muro sombreado respecto al caso base (CB47), por otra parte, los casos C4, C10, $\mathrm{C} 16, \mathrm{C} 22$ y $\mathrm{C} 28$, con TBS exterior de $35{ }^{\circ} \mathrm{C}$ y velocidad de viento de $6.5 \mathrm{~m} / \mathrm{s}$ muestran una disminución en temperatura mayor a aquellos casos con velocidad de viento de 4 y $1.5 \mathrm{~m} / \mathrm{s}$ (Gráfica 2). 


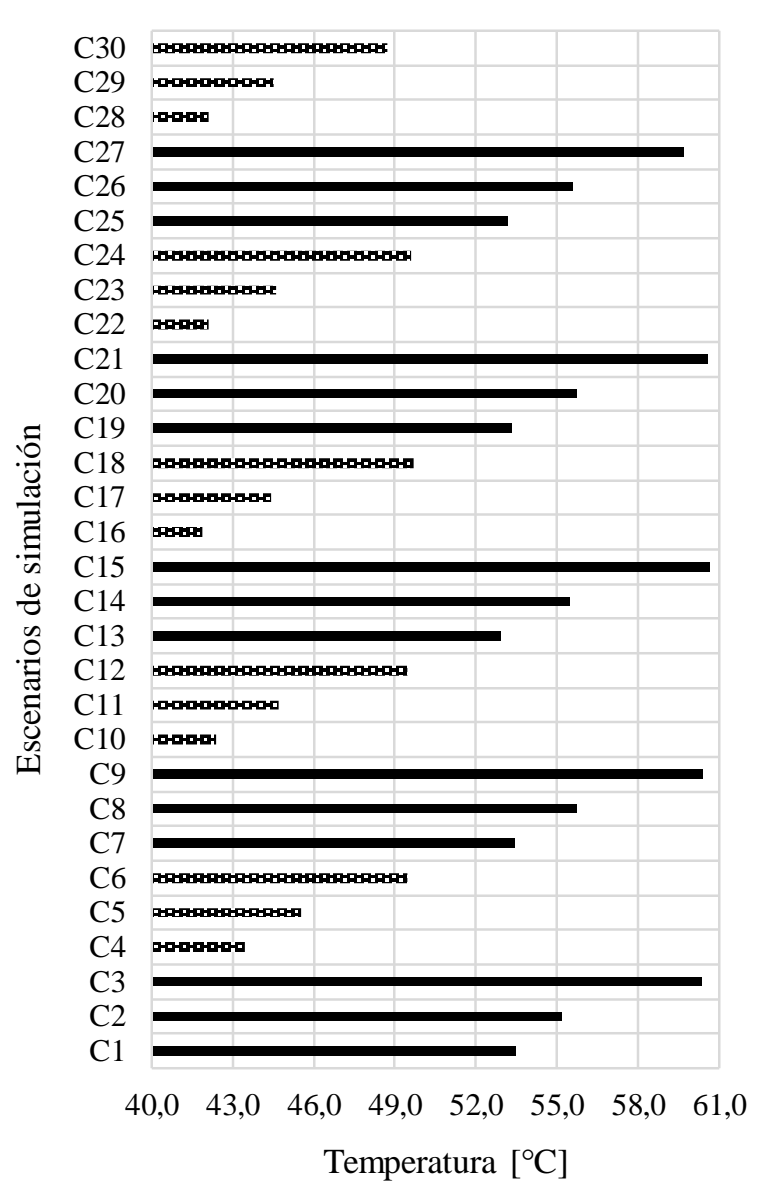

Gráfica 2 Temperatura superficial media en muro de block común de concreto

Se observa que, a mayor velocidad del aire de entrada, mejora el intercambio y la renovación de aire en el interior de la cavidad de aire, por lo cual, presenta una mayor reducción de temperatura.

4. Efecto del ancho de la cavidad de aire

La configuración geométrica de la fachada ventilada en este estudio tiene una variación paramétrica en el ancho de la cavidad de aire que va de $0.1 \mathrm{~m}$ a $0.5 \mathrm{~m}$. Las simulaciones muestran que la distancia entre los dos muros que conforman la cavidad de aire, con la caracterización de materiales que se utilizó en el estudio, presenta diferencias de temperatura superficial media de 0.4 a $1.6{ }^{\circ} \mathrm{C}$. Sin embargo, con respecto al caso base CB35 y CB47, muestran diferencias de temperatura de hasta 14.3-14.8 ${ }^{\circ} \mathrm{C}$.

En la gráfica 3 se observa que el caso con menor temperatura al centro de la cavidad de aire es el caso $\mathrm{C} 13$, con $0.3 \mathrm{~m}$ de espesor en la cavidad de aire, que respectivamente tiene la menor temperatura superficial media con respecto al CB47.
De igual manera en el caso C16 (con 0.3 $\mathrm{m}$ de espesor en la cavidad de aire), se observa la mayor reducción en el centro de la cavidad de aire con respecto al CB35, así como la menor temperatura superficial media con respecto al mismo caso base.

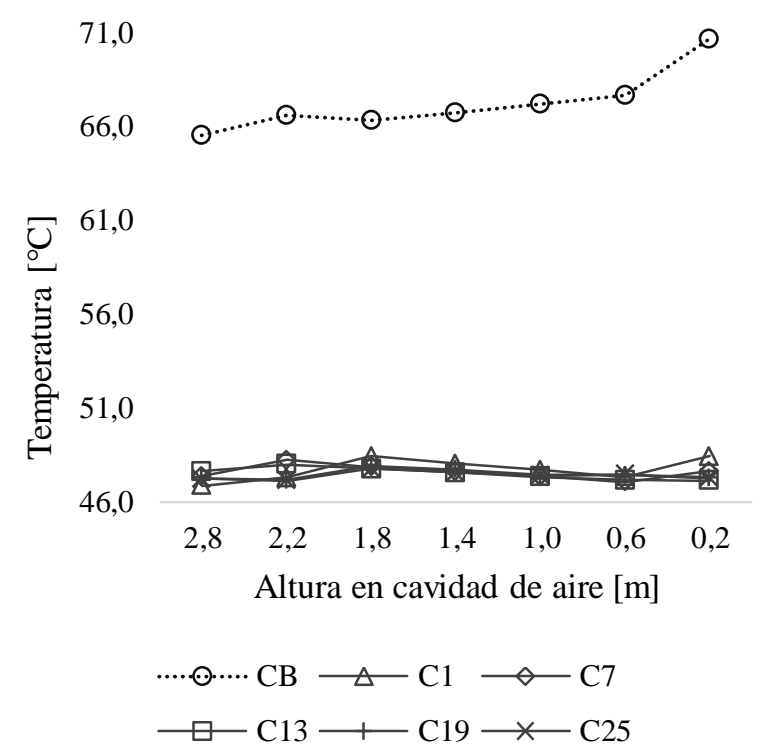

Gráfica 3 Temperatura al centro de la cavidad de aire para CB47

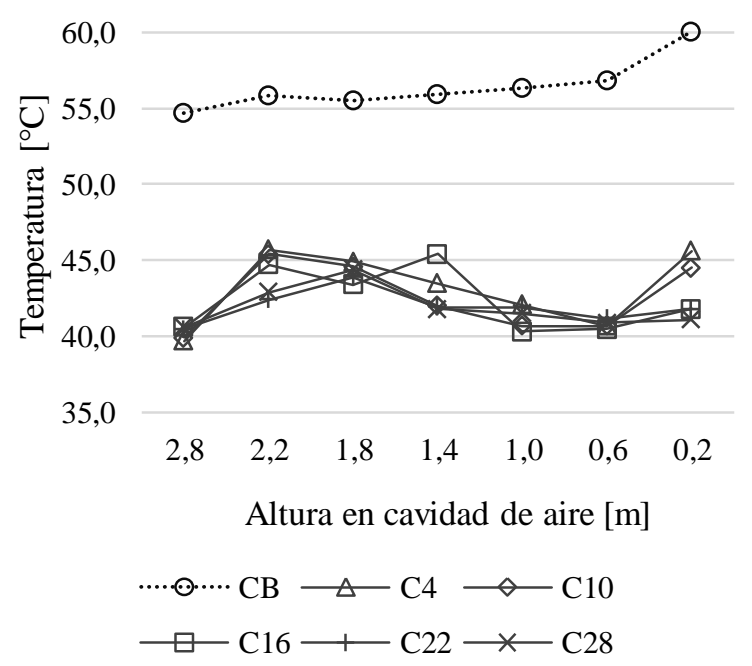

Gráfica 4 Temperatura al centro de la cavidad de aire para CB35

\section{Conclusiones}

Se observa de manera consistente con estudios anteriores, que un espesor mínimo de $0.1 \mathrm{~m}$ en la cavidad de aire tiene un efecto favorable sobre la temperatura superficial del muro en contacto con el ambiente interior y presenta un comportamiento estable a pesar del incremento en las dimensiones de la cavidad de aire, el mejor caso se presenta en el ancho de $0.3 \mathrm{~m}$.

SOTELO-SALAS, Cristina, ESPARZA-LÓPEZ, Carlos Javier y ESCOBAR-DEL POZO, Carlos. Comportamiento Térmico de fachada ventilada opaca en clima cálido seco extremo. Revista de Arquitectura y Diseño. 2019 
Adicionalmente, se determina que entre mayor sea la velocidad de viento, ésta genera una reducción mayor en la temperatura al centro de la cavidad de aire y en el muro de block común de concreto.

A pesar de que la temperatura dentro de la cavidad de aire es superior a la TBS exterior, la disminución con respecto a una fachada completamente expuesta a la radiación solar directa es de $14.3-14.8{ }^{\circ} \mathrm{C}$ como valor medio, y alcanza en algunos puntos $18{ }^{\circ} \mathrm{C}$ de diferencia, dicho comportamiento se considera alentador en la implementación de esta estrategia en edificaciones de clima cálido seco extremo.

Se observa que, de las tres variables estudiadas, la velocidad de viento es la que tiene mayor influencia sobre el comportamiento térmico de la fachada ventilada, con una diferencia de $4.72{ }^{\circ} \mathrm{C}$ entre los casos con velocidad de viento de $1.5 \mathrm{~m} / \mathrm{s}$ y $4 \mathrm{~m} / \mathrm{s}$, y una diferencia de $7.06{ }^{\circ} \mathrm{C}$ en los casos con velocidad de viento de $1.5 \mathrm{~m} / \mathrm{s}$ y los casos con $6.5 \mathrm{~m} / \mathrm{s}$.

El comportamiento térmico de la fachada ventilada opaca depende en gran medida de las condiciones ambientales al exterior de la edificación, y debido a que la velocidad de viento es la variable con mayor efecto sobre la reducción de temperatura, se sugieren estudios adicionales sobre criterios de diseño de la fachada ventilada que ayuden a incrementar la velocidad al interior de la cavidad de aire, ya sea la implementación de una chimenea solar aunada al cuerpo de la fachada ventilada, o la adición de un ventilador mecánico que garantice el recurso eólico necesario para la reducción de temperatura deseada.

Finalmente se considera que la fachada ventilada opaca es una estrategia de adecuación climática apropiada para edificaciones nuevas y existentes en clima cálido seco extremo, y debido a su comportamiento térmico, coadyuva en la reducción de la demanda de enfriamiento de dichas edificaciones, y por tanto disminuye su impacto ambiental.

\section{Agradecimiento}

Los autores agradecen al Programa Interinstitucional de Doctorado en Arquitectura y a la Universidad de Colima por su apoyo en la realización de este estudio, así como al Consejo Nacional de Ciencia y Tecnología por el apoyo económico. Se agradece a la Universidad Autónoma de Baja California por permitir el uso de los módulos experimentales de la Facultad de Arquitectura y Diseño.

\section{Referencias}

Barbosa, S., Ip, K., (2014). Perspectives of double skin façades for naturally ventilated buildings: A review. Renewable and Sustainable Energy Reviews, 40, 1019-1029.

Barbosa, S., Ip, K., Southall, R. (2015). Thermal comfort in naturally ventilated Buildings with double skin facade under tropical climate conditions: The influence of key design parameters. Energy and Buildings, 109, 397-406.

Bojórquez, G., Gómez-Azpeitia, L. G., GarcíaCueto, O. R., Ruiz-Torres, R. P., Luna, A. (2010). Temperatura neutral y rangos de confort térmico para exteriores, período cálido en clima cálido seco. Ambiente Construído, Porto Alegre, 10, (2), 133-146.

Esparza-López, C.J., Gómez-Amador, A., Gómez-Azpeitia, L.G., González-Cruz, E.M., Escobar, C. (2015). Desempeño térmico de tres dispositivos de enfriamiento evaporativo indirecto pasivo en clima cálido sub-húmedo. Palapa, III (2), 84-96.

Ghaffarianhoseini, A., Ghaffarianhoseini, A., Berardi, U., Tookey, J., Li, H. W., Kariminia, S. (2016). Exploring the advantages and challenges of double-skin facądes (DSFs). Renewable and Sustainable Energy Reviews, 60, 1052-1065.

Gutiérrez, M. T., Romero, R., Sotelo, C. (2014). Thermal energy impact of bioclimatic techniques applied to low-income housing in a hot dry climate. Energy Procedia. 57, 17431752 . 
Halawa, E., Ghaffarianhoseini, A., Ghaffarianhoseini, A., Trombley, J., Hassan, N., Baig, M., Yusoff, S., Ismail, M. A. (2018). A review on energy conscious designs of building façades in hot and humid climates: Lessons for (and from) Kuala Lumpur and Darwin. Renewable and Sustainable Energy Reviews, 82, 2147-2161.

Ibáñez-Puy, M. Vidaurre-Arbizu, M., SacristánFernández, J. A., Martín-Gómez, C. (2017). Opaque Ventilated Façades: Thermal and energy performance review. Renewable and Sustainable Energy Reviews, 79, 180-191.

Montazeri, H., Blocken, B., Hensen, J. L. M. (2015). CFD análisis of the impact of physical parameters on evaporative cooling by a mist spray system. Applied Thermal Engineering, 75, 608-622.

Sánchez, E., Rolando, A., Sant, R., Ayuso, L. (2016). Influence of natural ventilation due to buoyancy and heat transfer in the energy efficiency of a double skin facade building. Energy for Sustainable Development, 33, 139148.

Shameri, M.A., Alghoul, M. A., Sopian, K., Zain, M. F. M., Elayeb, O. (2011). Perspectives of double skin façade systems in buildings and energy saving. Renewable and Sustainable Energy Reviews, 15(3), 1468-1475.

Souza, L.C.O., Souza, H.A., Rodrigues, E.F., (2018). Experimental and numerical analysis of a naturally ventilated double-skin façade. Energy and Buildings, 165, 328-339.

Willmott C. J., (1981). On the validation of models. Physical Geography, 2 (2), 184-194. 